\title{
Early-type Galaxy Halo Dynamics inferred using the PN Spectrograph
}

\author{
N.G. Douglas ${ }^{1}$, A.J. Romanowsky ${ }^{2}$, K. Kuijken ${ }^{1,3}$, M.R. Merrifield ${ }^{2}$, \\ N.R. Napolitano ${ }^{1}$, M. Arnaboldi ${ }^{4}$, K.C. Freeman ${ }^{5}$, M. Capaccioli ${ }^{6}$, \\ O. Gerhard ${ }^{7}$ \\ ${ }^{1}$ Kapteyn Institute, Groningen; ${ }^{2}$ School of Physics 6 Astronomy, \\ University of Nottingham; ${ }^{3}$ University of Leiden; ${ }^{4}$ INAF-Osservatorio \\ Astronomico di Pino Torinese, Turin; ${ }^{5}$ RSAA, Mt. Stromlo and Siding \\ Spring Observatories; ${ }^{6}$ INAF-Osservatorio Astronomico di \\ Capodimonte, Naples; ${ }^{7}$ Astronomisches Institut, University of Basel
}

\begin{abstract}
A new instrument is providing crucial data with which to probe the structure of dark halos in elliptical galaxies
\end{abstract}

\section{Introduction}

Although evidence for dark halos in spiral galaxies is well-founded on kinematic measurements, similar studies in elliptical galaxies have not been so conclusive. One difficulty has been the lack of a suitable kinematic tracer at radii beyond the point at which integrated stellar spectroscopy becomes impractical. In the late 1980s the situation improved when planetary nebulae (PNe) were recognised as a powerful diagnostic, and since 2001 our team has been operating an instrument, the Planetary Nebula Spectrograph, which is specially designed for these observations. This results in a leap forward in detection efficiency and makes possible a range of new projects.

\section{The PN.Spectrograph}

The PN.S incorporates two highly efficient slitless spectrographs back-to-back. Via a common [OIII] filter this results in simultaneous images of the field in which the PNe are easily distinguished from stars. Their velocities can be derived from the relative shifts in their positions in the two images. This "Counter-Dispersed Imaging" technique is described in Douglas et al. (2002), along with other details of the project. Its value lies in the high optical efficiencies attained, in the absence of any astrometric requirements, and in the fact that it is a single-step procedure.

Our first project, currently in progress, is to examine the dark matter halos of apparently round, moderate-luminosity elliptical galaxies, too small to have been easily addressed by X-ray or gravitational-lensing analysis. Like most ellipticals, they do not produce much emission in neutral hydrogen either. Finally, conventional stellar spectroscopy does not probe far enough out, where dark-matter is expected to dominate. 

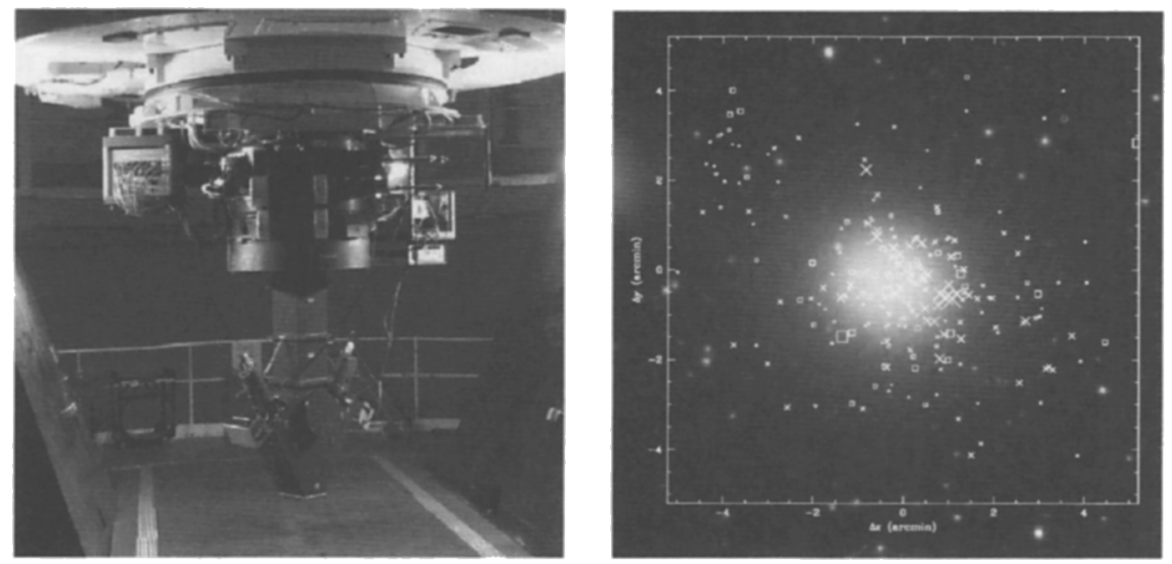

Figure 1. Left: the PN.S at the Cassegrain focal station of the William Herschel Telescope, which has built-in spectral lamps for calibrating the measured velocities. The PNS measures CDI images simultaneously by means of the two detectors shown here (photo courtesy of R.A. Hijmering). Right: the elliptical galaxy NGC 3379 with $230 \mathrm{PNe}$ superposed, the symbols indicating magnitude and sign of the velocity.

In the prevailing CDM model of galaxy formation the mass distribution is such that, over the region probed by our measurements, galaxies are expected to have a flat or slightly rising circular velocity profile (Navarro, Frenk, \& White 1996). This is in agreement with the asymptotically flat rotation curves of spiral galaxies and has also been borne out for the larger ellipticals (Gerhard et al. 2001). However the first three objects in our sample, NGC 3379, NGC 821 and NGC 4494, yielded the surprising result (though seen earlier in the case of NGC 3379 by Ciardullo, Jacoby \& Dejonghe 1993) that the velocity dispersion strongly declines with radius. With realistic assumptions about the isotropy of the velocity distribution this is indicative of a falling circular velocity profile and is almost consistent with there being no dark halo at all - see Romanowsky et al. elsewhere in these proceedings, and Romanowsky et al. (2003).

\section{References}

Ciardullo, R., Jacoby G.H., Dejonghe H.B. 1993, ApJ, 414, 454

Douglas, N. G., et al. 2002, PASP, 114, 1234

Gerhard, O., Kronawitter, A., Saglia, R. P. \& Bender, R. 2001, AJ, 121, 1936

Navarro, J. F., Frenk, C. S. \& White, S. D. M. 1996, ApJ, 462, 563

Romanowsky, A. J., et al. 2003, Science, 301, 1696 